\title{
Go-V vs. go-and-V in English: A case of constructional synonymy?
}

\author{
Stefanie Wulff
}

\begin{abstract}
The present paper deals with the double verb patterns go-and-V and go-V. While generative approaches have argued that the latter are truncated surface forms derived from the former, the present paper adds to the empirical evidence obtained so far, arguing in favor of the view that the two patterns be adequately conceived of as separate constructions (the notion of construction being defined in terms of construction grammar). On the basis of a large-scale corpus sample, statistical significance tests such as collostructional analysis and distinctive-collexemes analysis confirm that $g o-\mathrm{V}$ and go-and $-\mathrm{V}$ differ substantially in terms of the (aktionsart of the) verbs they attract; moreover, calculating the collexemic overlap between the two constructions, we find that the overlap of verbs shared between the two constructions is significantly smaller than would be expected according to a derivational view.
\end{abstract}

Keywords: fake coordination; synonymy; collocational overlap estimation; collostructional analysis; distinctive collexemes.

\section{Introduction}

The present paper deals with two superficially very similar double verb patterns in English, go-and-V and $g o-\mathrm{V}$, as exemplified in (1) and (2).

(1) Now, just keep polishing those glasses while I go and check the drinks.

(2) Go find the books and show me. 
In generative approaches, double verb constructions of the kind shown in (2) have been argued to be mere short versions derived from constructions like that in (1), while more functionally oriented researchers have argued that the two patterns behave differently with respect to their semantics, which rules out the possibility of the shorter pattern merely being a surface structure variant of the longer version. In particular, according to one of the central tenets of construction grammar, which is the theoretical framework adopted here, constructional synonymy is ruled out by the Principle of No Synonymy (cf. Goldberg 1995: 67, cf. Section 3).

The present paper approaches the question whether go-and-V and go- $\mathrm{V}$ are synonymous patterns from a corpus-linguistic perspective, arguing in favor of the hypothesis that go- $\mathrm{V}$ and go-and-V are not synonymous but rather constitute separate constructions (the notion of construction being defined in terms of construction grammar, cf. Section 3). In what follows, I will first provide a brief overview of previous approaches to go-(and)- $\mathrm{V}$ in Section 2. The hypotheses that can be derived from a derivational view will be tested on the basis of more than 5,000 instances of go-(and)-V obtained from the British National Corpus, the sampling and classification of which I will outline in Section 4. In Section 5, I will present the results produced by several statistical methods such as collocational overlap estimation, collostructional analysis, and distinctive collexeme analysis. The empirical evidence thereby obtained demonstrates that - contrary to the generative account $-g o-\mathrm{V}$ and go-and-V do in fact instantiate separate constructions.

\section{Previous approaches to go-and-V vs. go-V}

To begin with descriptive grammar books, while it is widely acknowledged that coordinated double verb patterns (mostly referred to as fake coordinations) display a variety of syntactic and semantic idiosyncrasies such as restrictions on the verb forms licensed to be inserted into the $V_{2}$-slot, their association with informal style ${ }^{1}$ and their often negative or derogatory connotation (cf. Quirk et al. 1985: 978-979; Huddleston and Pullum 2002: 1304), the relationship between go-and- $\mathrm{V}$ and go-V is rarely addressed directly. Eastwood (1994: 147) notes that go-V is the American English variant for British English go-and-V; however, this claim is proven wrong in view of the 454 examples found in the British National Corpus constituting a part of the data sample to be investigated here. 
Carden and Pesetzky (1979: 89) argue that $\mathrm{V}_{1}-\mathrm{V}_{2}$ constructions like go- $\mathrm{V}$ are derivates of the corresponding $\mathrm{V}_{1}$-and $-\mathrm{V}_{2}$ constructions, "presumably by a syntactic rule of Fake-and Deletion". They present three arguments to support their claim; however, they themselves have to concede that none of these arguments applies to go-(and)-V without confinements. Firstly, they claim that generally, both a $\mathrm{V}_{1}$-and- $\mathrm{V}_{2}$ pattern as well as its corresponding $\mathrm{V}_{1}-\mathrm{V}_{2}$ pattern share syntactic constraints such as the bare stem condition, which restricts the set of verb forms to be inserted into the patterns' verbal slots to non-inflected forms. However, Carden and Pesetzky (1979: 89) note that while this constraint holds for $g o-\mathrm{V}$, it does not hold for go-and$\mathrm{V}$; consider their examples in (3) and (4) respectively.

(3) a. *John went visit Harry yesterday.

b. He went and hit me.

Similarly, with respect to their second argument, saying that the semantics of $\mathrm{V}_{1}-\mathrm{V}_{2}$ patterns and $\mathrm{V}_{1}$-and $-\mathrm{V}_{2}$ patterns are identical, Carden and Pesetzky have to point out that while go-and-V has a possible "unexpected event reading" (consider [4a] and [4b], taken from Carden and Pesetzky [1979: 89]), go-V cannot encode such a meaning.

(4) a. ??As we had arranged, the President went and addressed the graduating class.

b. To our amazement, instead of addressing the graduating class, the President went and harangued the janitors.

Finally, Carden and Pesetzky point out that the set of verbs licensed in the $\mathrm{V}_{1}$ position of $\mathrm{V}_{1}-\mathrm{V}_{2}$ patterns constitute a subset of those of the $\mathrm{V}_{1}$-and- $\mathrm{V}_{2}$ patterns. Unfortunately, they do not provide any empirical evidence supporting this claim. To conclude, the failure of generative approaches to account for $g o-($ and $)-\mathrm{V}$ may be due to "a reluctance to think of a sequence that transgresses the boundary of two coordinated clauses as constituting a single grammatical construction" (cf. Hopper 2002: 146), since one of the fundamental commitments in generative grammar is a sharp dividing line between syntax and semantics.

Shopen (1971: 260) adopts a more functionally-oriented approach to $\mathrm{V}_{1}$ $\mathrm{V}_{2}$ constructions and argues against the view that $\mathrm{V}_{1}-\mathrm{V}_{2}$ constructions ${ }^{2}$ (to which he refers as quasi-modals) are to be considered merely "truncated surface variants of some other expression type". He points towards two 
semantic differences between $\mathrm{V}_{1}$-and $-\mathrm{V}_{2}$ constructions and their corresponding $\mathrm{V}_{1}-\mathrm{V}_{2}$ constructions to support his claim. First, the linkage between the two inserted verbs is much tighter in $\mathrm{V}_{1}-\mathrm{V}_{2}$ constructions than in $\mathrm{V}_{1}$-and- $\mathrm{V}_{2}$ constructions. Providing the examples in (5a) and $(5 \mathrm{~b})$, Shopen (1971: 260) argues that (5a) is unacceptable because the semantics of the two verbs go and leave are incompatible, while (5b) is acceptable due to the weaker linkage between the two verbs.

(5) a. *They deliberately go leave their wives behind.

b. They deliberately go and leave their wives behind.

Another difference between $\mathrm{V}_{1}-\mathrm{V}_{2}$ patterns and $\mathrm{V}_{1}$-and $-\mathrm{V}_{2}$ patterns Shopen identifies is that while go ordinarily allows both agential as well as nonagential interpretations (as in $g o-a n d-\mathrm{V}$ ), in the go-V pattern, the interpretation must be agential, which also speaks against the view that $g o-\mathrm{V}$ is only a short form of go-and-V. He illustrates his claim with the following examples.

(6) a. The trucks come and pick up the garbage every Monday.

b. The trucks come pick up the garbage every Monday.

(7) a. Pieces of drift wood come and wash up the shore.

b. *Pieces of drift wood come wash up the shore.

(8) a. The smoke fumes go and inebriate the people upstairs.

b. *The smokes go inebriate the people upstairs.

While the $(6 \mathrm{~b})$ is acceptable because the trucks can be associated with an agent, $(7 b) /(8 b)$ are unacceptable because the subjects pieces of drift wood and the smoke fumes do not license such an agential interpretation.

\section{Construction-based approaches to go-and-V}

In more recent theoretical frameworks, the strict dividing line between syntax and semantics is no longer upheld; instead, it is assumed that syntax and semantics are systematically intertwined to a considerable degree. One of these theoretical frameworks which appears to be especially well-suited for the explanation of structures like go- (and) $-\mathrm{V}$ and which is also adopted 
here is construction grammar. In construction grammar as developed by, among others, Goldberg $(1995,1996)$ and Lakoff $(1987)$, double verb patterns are assigned the status of a construction, the notion of construction being defined as follows:

A construction is ... a pairing of form with meaning/use such that some aspect of the form or some aspect of the meaning is not strictly predictable from the component parts or from other constructions already established to exist in the language. (Goldberg 1996: 68)

Several analyses have demonstrated that construction grammar can account for coordinate double verb constructions as in (1) in an elegant and straightforward way (cf. Lakoff 1986; Hopper 2002; Stefanowitsch 2000). Since neither of these analyses discusses the question of the relationship between go-and- $\mathrm{V}$ and go- $\mathrm{V}$ but exclusively focus on go-and- $\mathrm{V}$ and its status as a construction, I will not provide a detailed account of these analyses here; instead, the essentials of these analyses will be discussed in Section 5 to the extent deemed necessary in order to compare the semantics of go-and-V and $g o-\mathrm{V}$.

In spite of the fact that the relationship between go-and-V and go- $\mathrm{V}$ has not yet been addressed explicitly from a construction-based perspective, it is possible to derive the hypothesis that $g o-\mathrm{V}$ must be considered a separate construction from established theoretical commitments. According to one of the central tenets of Goldbergian construction grammar, the Principle of No Synonymy, "if two constructions are syntactically distinct, they must be semantically or pragmatically distinct" (Goldberg 1995: 67). So far, evidence for this principle comes from classic cases of "alternation" phenomena, such as dative movement, the load/spray alternation, the active/passive alternation, particle movement, and others. Each of these cases has been shown to consist of two distinct constructions, each with its own semantics and/or pragmatics (Goldberg 1995, 2002; Gries and Stefanowitsch 2004a). In all of these cases there is a difference in word order, often with additional lexical differences; however, there is little evidence so far concerning cases which, although one form has been argued to be derived from the other, have not been considered alternants on an equal footing, but where one structure is a shortened surface form of the other.

The present paper takes steps towards closing this gap. The following hypothesis follows from a derivational view: generally speaking, the two constructions should be semantically synonymous (in the absence of systematic semantic differences between the two patterns, the "unexpected 
event"-reading of go-and-V could be ignored as a solitary exception). Strictly speaking, it follows that the verbs inserted into the open slot of the go-V pattern should be identical to (or constitute a subset of) those of the go-and-V pattern. From a constructionist perspective, we would neither want to rule out that there is some degree of semantic overlap, since it is likely to assume that $g o-\mathrm{V}$ was actually once derived from go-and-V (this question being, however, beyond the scope of the present paper), so we can expect to find some semantic commonalities (particularly since a major part of the semantics of both constructions is contributed by the lexical semantics of go). However, according to the Principle of No Synonymy, we also expect that (i) there is a significant number of verbs in the go-V construction which do not occur in the go-and-V construction and vice versa, and that (ii) these two groups of verbs can be differentiated systematically with respect to their semantics, thereby legitimating the view that go-and-V and $g o-\mathrm{V}$ actually are individual constructions.

\section{Data and classification}

All instances of go- $\mathrm{V}$ and go-and- $\mathrm{V}$ were searched for in the British National Corpus. The initial sample comprising approximately 10,000 items was manually inspected in order to filter out about 5,000 instances of true coordination (and other patterns fitting the structural description). In accordance with the defining criteria for fake coordinations, the final sample did not include

- instances of go-PRT-and-V: these constitute constructions in their own right (cf. Stefanowitsch 2000);

- items in which a comma, full stop or any other punctuation mark intervened between $g o$ and the conjunction and, or $g o$ and $\mathrm{V}_{2}$ (in the case of go-V) respectively: these are not instances of go-(and)-V, since the punctuation mark signals the beginning of a new clause;

- items in which the conjunction and was not directly followed by $\mathrm{V}_{2}$, but by then, afterwards, or any other time adverbial: the presence of such an adverbial strongly indicates that the action denoted by go and the action denoted by $\mathrm{V}_{2}$ are interpreted sequentially, which renders these items cases of true coordination. 
The final sample size amounted to 5,320 instances of the go-and-V construction (comprised of 492 different $\mathrm{V}_{2}$ types) and 454 instances of the go$\mathrm{V}$ construction (comprised of 115 different $\mathrm{V}_{2}$ types).

If the constructions actually carry different meanings, this should primarily become evident in the choice of the only variable element in the constructional frame, $\mathrm{V}_{2}$. Accordingly, all $\mathrm{V}_{2} \mathrm{~S}$ were classified according to two different semantic classifications schemes. In a first step, the data were coded according to Vendler's (1967) situation types (aktionsarten); consider Table 1, which provides an overview.

Table 1. Situation types (adapted from Vendler 1967)

\begin{tabular}{ccc}
\hline & punctual & durative \\
\hline terminal & accomplishment & achievement \\
non-terminal & activity & state \\
\hline
\end{tabular}

These aspectual profiles constitute, of course, clausal properties when used in a specific context (cf. also Moens and Steedman [1988: 17]). Indeed, more often than not, only the context helped to unambiguously assign the tokens membership in one of the four classes. Therefore, the context is also provided in the following examples. As can be seen in Table 1, Vendler distinguishes four different situation types which are distinguishable along two dimensions, namely temporal extension (punctual vs. durative) and telicity (telic vs. atelic or, in Vendler's terminology, terminal vs. nonterminal). In other words, any action described by a verb can be distinguished according to (i) whether it extends in time or not and (ii) whether it has a culmination associated with it at which a change of state takes place or not. (9) to (12) provide examples from the present data sample.

(9) a. He can go and love some other girl and wed her if he can (state)

b. Why doesn't she go live round there or something (state)

(10) a. You've gone and achieved something (achievement)

b. Go find the books and show me (achievement)

(11) a. Shall I go and knock on her door and ask? (accomplishment)

b. Or you could do an Arnold Schwarzenegger, just go break the lock! (accomplishment)

(12) a. let's go and walk by the water's edge! (activity)

b. it would be nonsensical to get Paul Weller to go play live on a kids (activity) 
The second, more fine-grained semantic classification scheme employed here are Levin's (1993) verb classes. Her classification scheme is based on a systematic investigation of the different degree to which verbs do or do not undergo a variety of syntactic diathesis alternations. The primary hypothesis underlying this procedure is that these syntactic properties are semantically determined. As Levin notes,

[t]he assumption that the syntactic behavior of verbs is semantically determined gives rise to a powerful technique for investigating verb meaning that can be exploited in the development of a theory of lexical knowledge. If the distinctive behavior of verb classes with respect to diathesis alternations arises from their meaning, any class of verbs whose meanings pattern together with respect to diathesis alternations should be a semantically coherent class: its members should share at least some aspect of meaning. (Levin 1993: 14)

Levin's resulting classification scheme comprises 49 different classes covering a wide range of semantic classes such as verbs of putting, image creation verbs, verbs relating to the body, etc. These verb classes are further divided into sub-classes which have been ignored for the purpose of the present study. For reasons of space, not all of Levin's main verb classes can be enumerated here with examples; cf. section 4, where examples are given for those classes actually attested in the present data sample. The classification was based on Levin's own class assignment for each verb. 162 tokens [53 types] from the present data sample are not included in Levin's verb index. In order not to distort the picture unnecessarily, they were excluded from the data sample for this part of the analysis. If class membership was ambiguous (for instance with polysemous verbs like see), I determined the appropriate sense by considering the context of the item.

\section{Results}

5.1. The verbs of $g o-\mathrm{V}$ do not constitute a subset of the verbs of go-and-V

In order to test to what extent the two constructions are actually synonymous, the first analysis to be presented here is an ESCO (Estimation of Significant Collocate Overlap) analysis as proposed by Gries (2001). While Gries (2001) originally developed this technique in order to determine the semantic overlap of -ic and -ical adjectives, the method can be extended beyond the word level without difficulty given a construction grammar 
approach as adopted here, which postulates no fundamental difference in the constructional status of words and patterns of higher lexico-syntactic complexity. That is, Gries's (2001: 83) assumptions that “(i) word meanings can be differentiated on the basis of significant collocates and [...] (ii) we, thus, interpret a significant collocate of a word as one of its features" hold for more complex constructions as well. Accordingly, adapting Gries's method for go-and-V and $g o-\mathrm{V}$, the following three values enter into the computation of ESCO:

- the number of significant collexemes ${ }^{3}$ (i.e., features) that both go-and$\mathrm{V}$ and $g o-\mathrm{V}$ exhibit,

- the number of significant collexemes exhibited by go-and-V, but not go- $\mathrm{V}$, and

- the number of significant collexemes exhibited by go-V, but not goand $-\mathrm{V}$.

Figure 1 is a graphic representation of the proportions of these numbers.

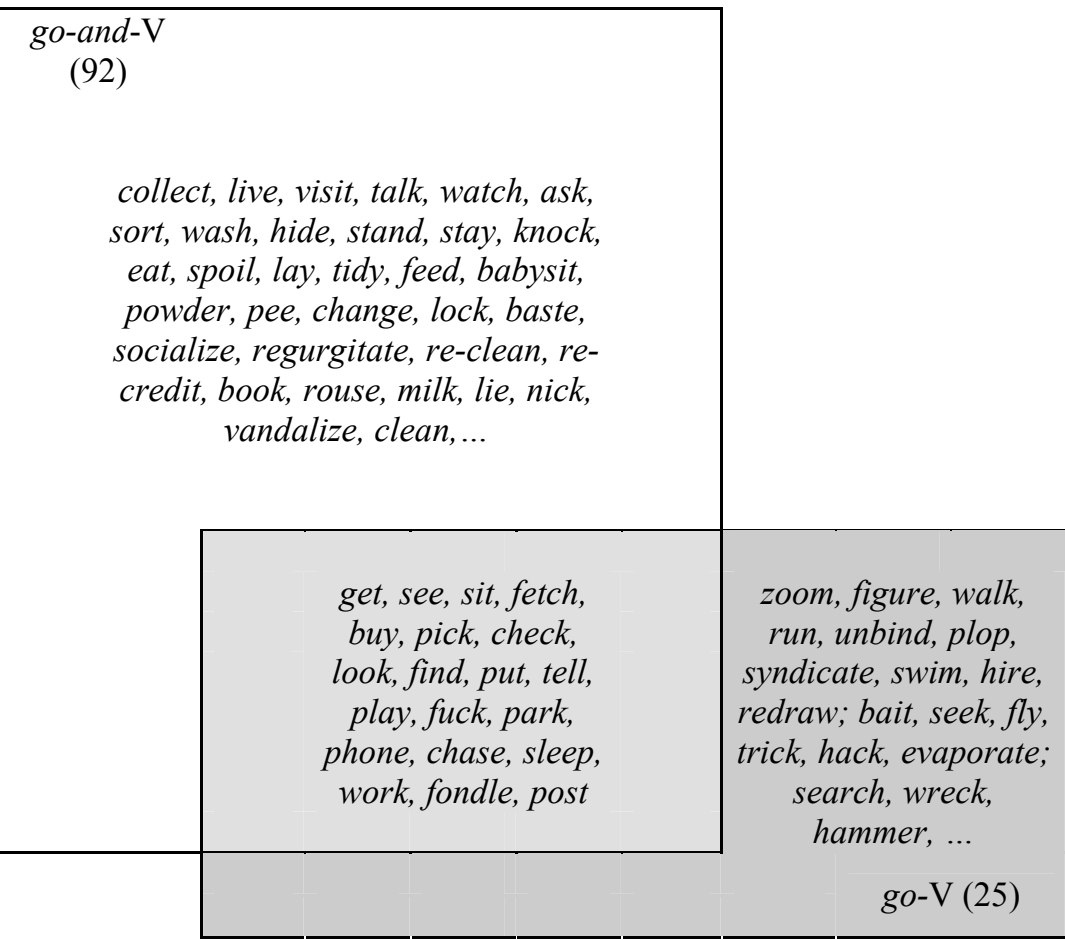

Figure 1. Significantly attracted collexeme set for go-and-V 
As is obvious from Figure 1, the overlap as determined by applying Gries's ESCO analysis is considerable. However, from the overlap percentages $(17.9 \%$ for go-and $-\mathrm{V}$ and $44.4 \%$ for $g o-\mathrm{V})$ it is completely unclear whether the overlap is significantly larger than would be expected on the basis of chance alone. To determine whether the overlap is in fact significant, two Monte Carlo simulations were carried out along the lines of Gries, Hampe, and Schönefeld (to appear). First, a list of all verb forms and their probabilities of occurrence in the BNC was obtained. ${ }^{4}$ Then 50,000 different samples of 45 verb forms (the number of significant collexemes of $g o-\mathrm{V}$ ) were drawn from that list where each verb forms chance of being corresponded to its relative frequency in the BNC. Then for each of the 50,000 samples it was counted how many of the 45 verb forms instantiated one of the 112 significant collexeme lemmas of go-and-V to determine how often this happened more than 20 times per sample. The same was done in the other constructional direction with 50,000 samples of 112 verb forms (the number of significant collexemes of go-and-V). As it turned out, an overlap of 20 was obtained only once in the (pooled) 100,000 random samples, which is why the overlap represented in Figure 2 must be considered highly significant: $\mathrm{p}=0.00001 .^{5}$

Note finally that even the direction of derivation that has been assumed by previous analyses is supported. Gries (2001: 105, n. 18) argued that, of the two items investigated, the one exhibiting higher overlap has "less an identity on its own", which is why one can plausibly assume the more strongly overlapping construction to be a derivative of the other. According to Figure 2, go-and-V has much more of an identity on its own, sharing only approximately $18 \%$ of its collexemes, which is why considering go-V a derivative receives some empirical support. On the basis of these findings, it comes as no surprise that much theoretical work has considered go$\mathrm{V}$ not only as highly similar to go-and- $\mathrm{V}$, but also a truncated derivative of the other. However, as I will show below, there are more differences between the two constructions than meet the eye at first.

\subsection{The semantics of the two constructions are not identical}

\subsubsection{Differences in aktionsart}

In order to determine how semantically similar the two patterns actually are, I carried out a so-called collostructional analysis along the lines of Stefanowitsch and Gries (2003); the computations (for all collostructional 
analysis discussed in this paper) were performed with Coll.analysis 3.2, an $\mathrm{R}$ script by, and freely available from, Stefan Th. Gries. By means of this method, it is possible to determine those lexemes in the slots of a construction which are strongly associated with the construction; these lexemes are also referred to as collexemes. In its paying tribute to the fact that the syntax of language is hierarchically structured, collostructional analysis goes beyond simply looking at the words directly preceding or following a word (i.e., the word's collocates), thereby enabling a more accurate description of the interdependency between particular lexemes and the syntactic structures they occur in. In order to test whether a lexeme L is significantly attracted or repelled by the construction $\mathrm{C}$, four frequency values are entered into a 2-by-2 matrix:

- the frequency of $\mathrm{L}$ in $\mathrm{C}$,

- the frequency of $\mathrm{L}$ in constructions that are not $\mathrm{C}$,

- the frequency of $\mathrm{C}$ where the free slot is filled with lexemes that are not $\mathrm{L}$, and

- the frequency of all constructions that are filled with lexemes that are not L.

The significance of the association between $\mathrm{L}$ and $\mathrm{C}$ is measured in terms of a Fisher exact test. Moreover, it is possible to identify which of these collexemes are either positively or negatively associated with the construction: if the co-occurrence frequency of $\mathrm{L}$ and $\mathrm{C}$ is higher than would be expected (given the general frequency of $\mathrm{L}$ and $\mathrm{C}$ ), $\mathrm{L}$ is attracted by $\mathrm{C}$; if, on the contrary, $\mathrm{L}$ fills the potential slot of $\mathrm{C}$ less frequently than would be expected, one can say that $\mathrm{L}$ is repelled by $\mathrm{C}$.

As already noted above, the only potential slot to be occupied in the go(and) $-\mathrm{V}$ constructions is the $\mathrm{V}_{2}$ slot, so semantic differences between the two constructions should be detectable by looking at the verbal collexemes.

Let us now turn to the results obtained by the collostructional analysis. First, we will have a look at the 25 most significantly attracted and repelled collexemes. ${ }^{6}$ Since it is comparatively difficult to find consistent semantic patterns by looking at the individual verbs alone, we will also consider the data when classified in terms of situation types and Levin's (1993) semantic verb classes.

To begin with go-and-V, Table 2 provides the 25 most significantly attracted/repelled collexemes of go-and-V. Table 3 displays the distribution of all significant collexemes (i.e., both the attracted as well as the repelled ones) with respect to situation types. 


\section{Stefanie Wulff}

Let us first consider the significantly attracted collexemes of go-and-V. As Table 2 in combination with Table 3 shows, verbs denoting accomplishments and achievements are predominant, e.g. get, fetch, check, and find, accounting for 2,274 $(1,328+946)$ out of 4,491 items containing a significant collexeme. Pure motion verbs, on the contrary, are rare: of the 2,053 items classified as activities, only 124 items are characterized as "physical" according to Levin's classification scheme. This predominance of telic verbs can be accounted for straightforwardly in terms of construction grammar: assuming that the aspect of "motion"/"dynamics" is an inherent part of the constructional meaning itself (primarily contributed by go), the $\mathrm{V}_{2}$ slot can be employed for encoding (compatible) additional information. Generally speaking, the fact that culmination verbs are so frequent among the significantly attracted collexemes of go-and-V does not come as a surprise if one assumes that the two verbs in the construction serve to encode a single event (cf. Lakoff 1986; Stefanowitsch 2000: 260): in an iconic linear ordering sequence, go describes the initiation of the event, and the $\mathrm{V}_{2}$ inserted describes the (way of) fulfillment of this action/event. ${ }^{7}$

Table 2. Collexemes most significantly attracted to/repelled by go-and-V

\begin{tabular}{cccc}
\hline $\begin{array}{c}\text { Attracted } \\
\text { collexemes }\end{array}$ & $\begin{array}{c}\text { Collostruction } \\
\text { strength }\end{array}$ & $\begin{array}{c}\text { Repelled } \\
\text { collexemes }\end{array}$ & $\begin{array}{c}\text { Collostruction } \\
\text { strength }\end{array}$ \\
\hline get & Inf(inity) & be & Inf \\
see & Inf & have & 55.879 \\
sit & 102.057 & go & 50.157 \\
fetch & 100.229 & say & 33.271 \\
buy & 68.134 & think & 33.245 \\
pick & 30.146 & come & 25.023 \\
check & 27.838 & use & 18.055 \\
look & 25.325 & become & 11.390 \\
find & 23.889 & follow & 9.087 \\
collect & 22.886 & believe & 6.579 \\
live & 21.892 & allow & 6.381 \\
visit & 21.810 & happen & 6.035 \\
talk & 20.639 & carry & 5.859 \\
put & 18.457 & continue & 5.690 \\
watch & 18.176 & involve & 5.328 \\
ask & 17.678 & suggest & 5.305 \\
tell & 15.269 & run & 5.256 \\
sort & 14.618 & offer & 5.099 \\
wash & 12.950 & keep & 5.026 \\
\hline
\end{tabular}




\begin{tabular}{cccc}
\hline play & 12.567 & set & 4.517 \\
hide & 9.838 & give & 4.438 \\
fuck & 8.886 & decide & 4.397 \\
stand & 8.239 & reach & 4.105 \\
stay & 7.607 & write & 3.613 \\
knock & 7.542 & pass & 3.612 \\
\hline
\end{tabular}

Table 3. Distribution of situation types in all tokens of significant collexemes for go-and- $\mathrm{V}$

\begin{tabular}{cc}
\hline Situation type & Token frequency \\
\hline Activity & $2,049(45.6 \%)$ \\
Accomplishment & $1,328(29.6 \%)$ \\
Achievement & $946(21.1 \%)$ \\
state & $168(3.7 \%)$ \\
Column totals & $4,491(100 \%)$ \\
\hline
\end{tabular}

Considering the most strongly repelled collexemes in Table 2 , we find that the two verbs most strongly repelled by go-and-V are be and have, both of which denote states (other stative verbs among the repelled collexemes are, e.g., think, believe and keep, amounting to 168 out of 4,491 items including a significant collexeme). So although stative verbs do occur in the construction, which contradicts previous analyses claiming that this is impossible (cf. Hopper 2002), stative verbs occur much less frequently in the construction than their general corpus frequency would lead us to expect. As a matter of fact, this ties in well with Stefanowitsch's (2000: 261) observation that the essential semantic contribution made by go in the go-and-V construction is "motion along a path", i.e. a dynamic feature. This leads us to expect that $V_{2}$ will also be inherently dynamic or at least lend itself to such an interpretation if it is inserted into the construction. Stative verbs, on the contrary, should be disfavored by the construction because they are incompatible with the constructional semantics, and this is exactly what the collostructional analysis tells us (cf. Goldberg 1995: 50-51).

So what about the cases where the verb actually is stative? A construction-based approach can also account for these seemingly contradicting cases. As Stefanowitsch (2000: 261) notes, the semantics of the construction and the lexical semantics of the verb blend into each other such that not only the lexical semantics of the verb adds new information to the con- 


\section{Stefanie Wulff}

struction as a whole, but also vice versa such that the way in which the verb's meaning will be interpreted is strongly influenced by the constructional frame in which it occurs (cf. Stefanowitsch 2000: 261). Consequently, even verbs that are interpreted as stative in the default case can be inserted into the construction given that a more dynamic reading is possible, even if it is one very remote from the verb's core meaning. How the choice of one sense of a polysemous verb is motivated by the constructional semantics is nicely illustrated by the most strongly attracted collexeme see: as opposed to its prototypical perceptive meaning (which is relatively stative in nature), in the go-and-V construction, it takes on the more dynamic meaning 'watch'/'visit'; consider (13) and (14).

\section{(13) I might go and see Aunt Violet}

(14) But, we pleaded, can we go and see a proper team like Sunderland next time

Similarly, the third most strongly attracted collexeme, sit, the core meaning of which is also non-dynamic, yields an event-like interpretation in the goand-V construction, as illustrated in (15) and (16).

... middle-aged people that could go and sit in there for a drink

(16) Or shall we go and sit at the café?

So far, the results of the collostructional analysis largely confirm already established claims about the semantics of the go-and-V construction. However, the analysis also reveals some aspects about the usage of this construction that have hitherto not been noticed. One interesting aspect which, admittedly, can only be detected if one considers all 112 significantly attracted collexemes of the construction - is that it is very frequently employed with verbs denoting cleaning and/or bodily hygiene such as clean, scrub, shower, powder, and wipe; these usages completely lack the derogatory or foolish connotation that typically characterizes a large part of the examples. Consider two typical examples in (17) and (18).

(17) go and tidy your rubbish up darling

(18) I think I'll go and wash my hair 
This result is particularly interesting when being combined with the ESCO results (cf. Figure 1), which show that the verbs relating to (bodily) hygiene are all among the group of verbs which exclusively occur with go-and-V; this points towards the fact that go-and-V in fact has a specified semantic profile that is different from the one of $g o-\mathrm{V}$.

Let us now turn to the significant collexemes of $g o-\mathrm{V}$ in Table 4 and their distribution with respect to situation types as shown in Table $5 .{ }^{8}$

Table 4. Collexemes most significantly attracted to/repelled by go-V

\begin{tabular}{cccc}
\hline $\begin{array}{c}\text { Attracted } \\
\text { collexemes }\end{array}$ & $\begin{array}{c}\text { Collostruction } \\
\text { strength }\end{array}$ & $\begin{array}{c}\text { Repelled } \\
\text { collexemes }\end{array}$ & $\begin{array}{c}\text { Collostruction } \\
\text { strength }\end{array}$ \\
\hline get & 48.177 & have & 18.330 \\
fuck & 19.399 & say & 3.687 \\
see & 14.769 & do & 1.949 \\
fetch & 11.522 & & \\
work & 6.950 & & \\
zoom & 6.949 & & \\
tell & 6.924 & & \\
buy & 6.626 & & \\
look & 6.480 & & \\
sleep & 4.834 & & \\
figure & 4.736 & & \\
walk & 4.649 & & \\
park & 4.140 & & \\
phone & 3.674 & & \\
run & 3.448 & & \\
check & 3.240 & & \\
unbind & 3.016 & & \\
play & 2.972 & & \\
find & 2.749 & & \\
plop & 2.724 & & \\
post & 2.719 & & \\
syndicate & 2.658 & & \\
chase & 2.636 & & \\
swim & 2.445 & & \\
fondle & 2.420 & & \\
\hline & & & \\
& & & \\
& & & \\
\end{tabular}


Table 5. Distribution of situation types in all tokens of significant collexemes for go- $\mathrm{V}$

\begin{tabular}{cc}
\hline Situation type & Token frequency \\
\hline activity & $180(54.4 \%)$ \\
accomplishment & $44(13.3 \%)$ \\
achievement & $95(28.7 \%)$ \\
state & $12(3.6 \%)$ \\
Column totals & $331(100 \%)$ \\
\hline
\end{tabular}

Interpreting Table 5, we find that a substantial number of $g o-\mathrm{V}$ 's attracted collexemes $(44+95 / 331)$ belongs to the class of accomplishment and achievement verbs such as check, fetch, find, and get. However, the share of process verbs which are atelic is also considerably high. As for go-and- $\mathrm{V}$, the collexemes most strongly repelled belong to the situation type class of states (cf. Table 3). Once we focus exclusively on the opposition pair of accomplishments/achievements and atelic activities/states, an interesting picture emerges; consider Table 6 , which provides the observed frequencies (as well as the expected frequencies in parentheses) for these verb classes.

Table 6. Number of telic vs. atelic verbs for go-and-V

\begin{tabular}{cccc}
\hline & Telic verbs & Atelic verbs & Row totals \\
\hline go-and-V & $2,274(2,247)$ & $2,217(2,244)$ & 4,491 \\
go-V & $139(166)$ & $192(165)$ & 331 \\
Column totals & 2,413 & 2,409 & 4,822 \\
\hline
\end{tabular}

A Fisher exact test shows that go-V takes verbs denoting accomplishments/achievements less often than would be expected; this tendency is highly significant $\left(\mathrm{p}_{\text {Fisher exact }}<.01\right)$.

To conclude, the results of the collostructional analysis and the interpretation of the results in terms of situation types has shown that the two constructions are not semantically identical because the proportions of verb types they take differ notably. Moreover, these differences are not random, but can be described in a systematic pattern: while go-and-V prefers telic verbs, go-V preferably takes atelic process verbs. However, the two constructions also share features, e.g., both repel stative verbs. 


\subsubsection{Differences in terms of Levin's (1993) verb classes}

In order to pinpoint the semantic differences between the two constructions, the classification of the verbs according to situations types obviously is too coarse-grained and does not lead us any further. Consider Table 7, which provides an overview of which of Levin's (1993) verb classes were instantiated by how many significant collexemes (of both constructions). For each verb class, at least one example of a significant collexeme is given. The first of the two frequency values given in each cell is the observed number of items among the significant collexemes of the respective construction for each semantic class; the second value in brackets is the expected frequency. ${ }^{9}$

A chi-square test shows that the overall distribution is significant $\left(\chi^{2}=301.46 ; \mathrm{df}=32 ; \mathrm{p}<.001 * * * 10\right)$. Those cells which are responsible for this general significance, i.e., so-called contributions to chi-square, are printed in bold face. Finally, the plus or minus signs at the right top corners indicate whether the observed frequency is significantly higher or lower than expected.

The most interesting fact to be derived from Table 7 is that go-V significantly more often attracts verbs classified as relating to the body, motion and perception (I leave aside the statistical significance for the class of destroy verbs because only a single verb yields a significant value). That is to say, go-V favors verbs which, in their most typical sense, relate to physical behaviour and activity. In other words, whereas in the go-and-V construction, the dynamic aspect is part of the constructional semantics and motivates the choice of a correspondingly dynamic sense of a polysemous verb - even if this sense is a remote one, it appears that $g o-\mathrm{V}$ rather prefers verbs which already carry this semantic property in their more prototypical, default senses. 
Table 7. Distribution of Levin's (1993) verb classes

\begin{tabular}{|c|c|c|c|}
\hline Verb class & Example & go-and-V & $g o-\mathrm{V}$ \\
\hline do & do & $247(244)$ & $14(17)$ \\
\hline $\begin{array}{c}\text { verbs of (dis)appearance / } \\
\text { occurrence }\end{array}$ & come, die & $20(19)$ & - (1) \\
\hline aspectual verbs & start, continue & $12(11)$ & - (1) \\
\hline bear & bear & $4(4)$ & $-(-)$ \\
\hline body verbs & sleep, smack & $17(21)$ & $6^{\oplus}(2)$ \\
\hline calve verbs & foal & $1(1)$ & $-(-)$ \\
\hline verbs of change & fetch, buy & 1,648 & $116(115)$ \\
\hline & freshen, & $9(1$ & $2(1)$ \\
\hline verbs of combinit & baste, lock & $12(11)$ & - (1) \\
\hline $\mathrm{co}$ & ask, tell & 359 & $24(25)$ \\
\hline $\mathrm{cc}$ & hide & 20 & $-(1)$ \\
\hline verbs of & knock, smash & $18(17)$ & $-(1)$ \\
\hline verbs of & change, prep & $261(261)$ & $18(18)$ \\
\hline & nick & 3 & $-(-)$ \\
\hline verbs o & wish & $1(1)$ & $-(-)$ \\
\hline destrc & wreck & $-(1)$ & $\mathbf{1}^{\oplus}(-)$ \\
\hline $\mathrm{ve}$ & gurgle & $1(1)$ & $-(-)$ \\
\hline & live, stay & 103 & $-\ominus(7)$ \\
\hline verbs c & powder, & 38( & $-(2)$ \\
\hline & draw, $w$ & $12(11)$ & $-(1)$ \\
\hline & eat, feed & $33(31)$ & $-(2)$ \\
\hline mea & fit & $1(1)$ & $-(-)$ \\
\hline motion verbs & jump, run & $54(74)$ & $25^{\oplus}(5)$ \\
\hline perception verbs & hear, see & $188(229)$ & $\mathbf{5 7}^{\oplus}(16)$ \\
\hline verbs of assumin & kneel, sit & 237 (227) & $6(16)$ \\
\hline predicative & discover, pro & $678(637)$ & $3^{\ominus}(44)$ \\
\hline & courage, & 5( & $1(-)$ \\
\hline verbs & lay, stick & $133(136)$ & $12(9)$ \\
\hline & clean, wipe & $24(22)$ & $-(2)$ \\
\hline ver & check, inspect & $99(102)$ & $10(7)$ \\
\hline verbs of sending/carrying & post, send & $8(9)$ & $2(1)$ \\
\hline $\begin{array}{c}\text { verbs of } \\
\text { separating/disassembling }\end{array}$ & unhook, unlace & $2(2)$ & $-(-)$ \\
\hline verbs of social interaction & hobnob, visit & $109(108)$ & 7 \\
\hline Column totals & & 4,357 & 304 \\
\hline
\end{tabular}




\subsubsection{Distinctive collexeme analysis}

The third corpus-linguistic method applied to the data is a so-called distinctive collexeme analysis, which measures the dissimilarity of semantically similar constructions on the basis of their significant collexemes (cf. Gries and Stefanowitsch's [2004a, 2004b] extension of Church et al.'s [1991] technique to investigate distinctive collocates). Accordingly, the distinctive collexemes of two constructions are those words which distinguish best between the two constructions. Four frequency values enter into the computation of a collexeme's distinctiveness:

- the lemma frequency of the collexeme in construction A,

- the collexeme's lemma frequency in construction B,

- the (added) frequencies of all other collexemes with construction A, and

- the (added) frequencies of all other collexemes with construction B.

These numbers are entered into a 2-by-2 matrix and are subjected to a Fisher exact test. A corresponding p-value tells us how distinctive a collexeme is for a particular construction; the smaller the value, the higher the collexeme's distinctiveness.

Let us have a look at the results obtained by the distinctive collexeme analysis and see in how far they support the assumptions made about the difference between the two constructions made so far. Table 8 displays all significant distinctive collexemes for go-and-V vs. go-V; for both constructions, we find the distinctive collexemes (in decreasing order of their distinctiveness) in the left-hand column, together with the ratio of their frequency in the constructions. For instance, the ratio of 357:8 of the collexeme have means that have occurs 357 times in the go-and-V construction, but only 8 times in the go-V construction, which renders have highly distinctive for go-and-V. In the right-hand column, the corresponding $\mathrm{p}$-values for each collexeme are given.

As Table 8 shows, the distinctive-collexeme analysis unanimously supports the results gained of the collostructional analysis: with the exception of talk, the overwhelming majority of the distinctive collexemes for goand $-\mathrm{V}$ are verbs which may either have a stative or, as has been argued above, a dynamic reading which is selected if the verb is inserted into the construction (have, sit, see, stand). The distinctive collexemes for go- $\mathrm{V}$, on the other hand, are mostly motion verbs (run, fuck, walk, fly, swim) or verbs 
which imply (ongoing physical) activity (work, seek). To conclude, the distinctive-collexemes analysis supports the hypothesis about the semantic inequality of go-and- $\mathrm{V}$ and $g o-\mathrm{V}$.

Table 8. Distinctive collexemes for go-and $-\mathrm{V}$ and $g_{0}-\mathrm{V}$

\begin{tabular}{cccc}
\hline \multicolumn{2}{c}{ go-and-V } & \multicolumn{2}{c}{ go-V } \\
Collexeme & Distinctiveness & Collexeme & Distinctiveness \\
\hline have (357:8) & 5.769 & run $(4: 8)$ & 6.294 \\
sit $(162: 5)$ & 2.134 & fuck $(10: 10)$ & 6.138 \\
see $(675: 40)$ & 2.090 & work $(48: 16)$ & 4.640 \\
stand (46:0) & 1.640 & walk $(8: 7)$ & 4.188 \\
talk $(67: 1)$ & 1.593 & seek $(1: 4)$ & 3.754 \\
collect (37:0) & 1.318 & figure $(0: 3)$ & 3.318 \\
& & zoom $(0: 3)$ & 3.318 \\
& & fly $(1: 3)$ & 2.742 \\
& & give $(38: 10)$ & 2.458 \\
& & sleep $(10: 5)$ & 2.345 \\
& & swim $(0: 2)$ & 2.211 \\
& & tell $(101: 17)$ & 2.211 \\
& & pass $(1: 2)$ & 1.993 \\
& & park $(8: 3)$ & 1.757 \\
& & phone $(8: 3)$ & 1.307 \\
\hline
\end{tabular}

Moreover, bringing together the results obtained from the collostructional analyses and the ESCO analysis, we find that the group of collexemes which go-V does not share with go-and-V are exactly the ones which the collostruction and distinctive collexeme analyses identified as the ones best representing its constructional semantics, namely process verbs.

These findings, although they do not strictly preclude the possibility that go- $\mathrm{V}$ and go-and- $\mathrm{V}$ are related constructions or even that the shorter one was once derived from the longer one, nevertheless cast serious doubt on the hypothesis that go- $\mathrm{V}$ simply is a truncated form of go-and-V. If this were the case, one would expect that the core constructional semantics of go-and-V, namely its dynamism and event-like reading, are inherited by the more specific construction, go- $\mathrm{V}$. However, in go-V constructions, the constructional semantics are reduced to "initiation of action/event", with a strong focus on the procedural aspects of this action or event, without it necessarily being brought to an (foreseen) end. This also ties in well with Shopen's (1971) above-mentioned observation that the linkage between $V_{1}$ and $\mathrm{V}_{2}$ is stronger in the constructions lacking a conjunction in comparison 
to those including and. Moreover, the auxiliary-like function of $g o$ as noted by Stefanowitsch (2000) appears to be even stronger for $g o-\mathrm{V}$ than for goand $-\mathrm{V}$, which is also reflected in its closer position to $\mathrm{V}_{2}$.

\section{Conclusion}

This paper demonstrates that - contrary to the generative account $-g o-\mathrm{V}$ and go-and $-\mathrm{V}$ do in fact instantiate separate constructions. That is, go- $\mathrm{V}$ is not a truncated surface variant which means the same as its mother structure, as generative approaches like the Fake and Deletion rule would imply, but the shortening process has also led to a change, or, more precisely, a reduction and re-focusing, in meaning. That is, while whatever action is denoted by go-and-V gains an event-like interpretation and is meant to embrace the whole sequence cascade of a typical event with a beginning and an end, the meaning of $g o-\mathrm{V}$ only denotes the initiation of an action and is inherently atelic, which invites process verbs to occupy the $\mathrm{V}_{2}$ slot. While the Principle of No Synonymy has formerly been tested only on the basis of classic 'alternation' phenomena, the results of the present analysis show that it holds also for constructions where one structure is considered a shortened surface form of the other, thereby taking a first step towards closing an empirical gap.

Moreover, this paper has made two strong methodological points. Firstly, it has emphasized the need for large-scale and representative data samples as well as an exhaustive examination of these data in order to yield a comprehensive picture of the actual range of usage of a pattern. The fact that former analyses (irrespective of their theoretical orientation) concluded that go-and-V and go-V must be synonymous comes as no surprise once we have a look at the collexemic overlap estimation of the two constructions, which nicely illustrated how misleading it may be to consider only the most frequently occurring attestations of a particular pattern. Secondly, the paper has shown how semantic differences between near-synonymous constructions like go-V and go-and-V can only be identified in combining several corpus-linguistic methods: while the ESCO analysis revealed that indeed a range of verbs occur in both constructions, the collostructional analyses revealed how those verbs which are not shared between the two constructions form fairly homogenous semantic groups in terms of aktionsart and Levin's (1993) semantic classes. Taken together, we find that that these semantic differences are neither diffuse nor quantitatively marginal, but highly systematic and quantitatively significant. 
Last but not least, in applying the corpus-linguistic techniques developed by Gries and Stefanowitsch to a research question other than the one(s) they were originally designed for, namely the field of constructional synonymy, the present paper may serve as a model how future research in related fields such as polysemy, word sense disambiguation, etc. can benefit from applying these methods, too.

\section{Notes}

1. With respect to the claim that go-and-V is strongly associated with informal style, I would like to point out that in the present data sample, $54 \%(2,876$ items) of the go-and-V data as well as $56 \%$ (253 items) of the go- $\mathrm{V}$ data were obtained from the spoken subcorpus of the BNC, so it does not appear that the constructions are actually only associated with informal and colloquial register, but they are just as frequently employed in written language.

2. It has to be noted here that with the exception of the work of Stefanowitsch (2000), all other approaches to $\mathrm{V}_{1}-($ and $)-\mathrm{V}_{2}$ constructions discussed here do not employ the term construction in the sense of construction grammar, but rather in a traditional sense.

3. A collexeme is any verb inserted into the $\mathrm{V}_{2}$-slot of go- $\mathrm{V} /$ go-and- $\mathrm{V}$ which is significantly associated with the respective construction; cf. Section 5.2, where the method(s) employed for obtaining each construction's significant collexemes is explained in more detail..

4. I included only those forms which were unambiguously tagged as verbs or had a portmanteau tag with the verb tag first.

5. One final comment is in order here. One might object to the above kind of simulation since Figure 2 was concerned with significant collexemes while the Monte Carlo simulations did not - it counted collexemes only irrespective of whether they had a significant association to any construction. However, performing the test as reported here should be an even more stringent test of the overlap hypothesis because the percentage of overlapping significant collexemes is much smaller than those of all collexemes $(17.4 \%$ for go-and-V and $74.7 \%$ for $g o-\mathrm{V}$ ), so if the simulated overlap figures do not even reach the lower percentages of the significant collexemes, they cannot possibly reach those of all collexemes.

6. For reasons of space, I decided to present only the first 25 most significantly attracted/repelled collexemes for each construction. The overall number of significant collexemes amounts to 112 attracted/76 repelled collexemes for go-and- $\mathrm{V}$ and 45 attracted / 3 repelled for $g o-\mathrm{V}$. 
7. In the publications introducing collostructional analysis, the strength of association and repulsion is measured by the p-value of a Fisher-Yates exact test. In the present work, I use the negative logarithm to the base 10 of this p-value because the resulting numbers are easier to interpret. Accordingly, to reach a $5 \%$ level of significance, the value must be higher than 1.3 , and a difference of one between two verbs' collostructional strength corresponds to a difference of one order of magnitude. For cases like get and see in Table 2 where the p-values are so small that the default settings of Collanalysis 3 report them as infinite, I determined the actual ranking of the verbs by setting the program to use log-likelihood values (1,884.34 and 1,494.72 respectively) for ranking instead.

8. The fact that go-V has fewer significant collexemes than go-and-V can be seen as a mirror image of the general frequency of these constructions, $g o-\mathrm{V}$ being much less frequent than go-and-V (454 vs. 5,320 tokens in the present data sample), as well as the correspondingly smaller number of different $V_{2}$ types occurring in the respective constructions (115 vs. 491 types).

9. Do and bear are not assigned membership in any particular class but are treated separately in Levin's (1993) analysis, which is why they are itemized here.

10. Strictly speaking, one cannot use the chi-square test for this table since more than $20 \%$ of the expected frequencies are smaller than five. The exact alternative which is not sensitive to such distributions, the Freeman-Halton test, proved to be computationally too expensive for such a large sample. Therefore, I conducted two Monte-Carlo-like simulations of multinomial tests to assess the degree of deviation between observed and expected cell frequencies (using Multnomtest 1.0, an R script by, and available from, Stefan Th. Gries). In the first simulation, I tested whether the observed distribution of verb classes for go-and- $\mathrm{V}$ could be obtained when the expected distribution is that following from the $g o-\mathrm{V}$ verb classes. Thus, I drew 100,000 random samples of 4,491 constructions with the input probabilities of the $g o-\mathrm{V}$ distribution and counted how often I obtained a distribution that deviated from the observed one as extremely or even more extremely. The second simulation was performed in the reverse direction, asking how often the distribution of verb classes for $g o-\mathrm{V}$ was obtained when the expected frequencies followed from the go-and-V distribution. In both cases, i.e. all 200,000 simulations, not a single such distribution was obtained, which reflects that the distributions of verb classes in both constructions differ very strongly. 


\section{References}

Carden, Guy and David Pesetzky

1979 Double-verb constructions, markedness, and a fake co-ordination. CLS 13: 82-92.

Church, Kenneth W., William Gale, Patrick Hanks, and Donald Hindle

1991 Using statistics in lexical analysis. In: Uri Zernik (ed.), Lexical acquisition: Exploiting on-line resources to build up a lexicon, 115164. Hillsdale, NJ: Lawrence Erlbaum.

Eastwood, John

1994 Oxford Guide to English Grammar. Oxford: Oxford University Press.

Goldberg, Adele E.

1995 Constructions. a construction grammar approach to argument structure. Chicago/London: University of Chicago Press.

1996 Construction grammar. In: Keith Brown and Jim Miller (eds.), Concise Encyclopedia of Syntactic Theories, 68-71. Oxford: Pergamon.

2002 Surface generalizations: An alternative to alternations. Cognitive Linguistics 13 (3): 327-356.

Gries, Stefan Th.

2001 A corpus-linguistic analysis of -ic and -ical adjectives. ICAME Journal 25: 65-108.

to appear New perspectives on old alternations. CLS 39.

Gries, Stefan Th., Beate Hampe, and Doris Schönefeld

in press Converging evidence II: More on the association of verbs and constructions. In: John Newman and Sally Rice (eds.). Empirical and Experimental Methods in Cognitive/Functional Research. Stanford, CA: CSLI Publications.

Gries, Stefan Th. and Anatol Stefanowitsch

2004a Extending collostructional analysis: A corpus-based perspective on “alternations". International Journal of Corpus Linguistics 9 (1): 97129.

2004b Covarying collexemes in the into-causative. In: Michel Achard and Suzanne Kemmer (eds.), Language, Culture, and Mind, 225-236. Stanford, CA: CSLI Publications.

Hopper, Paul

2002 Hendiadys and auxiliation in English. In: Joan L. Bybee and Michael Noonan (eds.), Complex sentences in grammar and discourse: Essays in honor of Sandra A. Thompson, 145-173. Amsterdam/Philadelphia: John Benjamins. 
Huddleston, Rodney and Geoffrey K. Pullum

2002 The Cambridge Grammar of the English Language. Cambridge: Cambridge University Press.

Jaeggli, Osvaldo A. and Nina M. Hyams

1993 On the independence and interdependence of syntactic and morphological properties: English aspectual come and go. Natural Language and Linguistic Theory 11: 313-346.

Lakoff, George

1987 Women, Fire, and Dangerous Things. What Categories Reveal about the Mind. Chicago/London: University of Chicago Press.

Levin, Beth

1993 English Verb Classes and Alternations. A Preliminary Investigation. Chicago/London: Chicago University Press.

Moens, Marc and Mark Steedman

1988 Temporal ontology and temporal reference. Computational Linguistics 14 (2): 15-28.

Quirk, Randolph, Sidney Greenbaum, Geoffrey Leech, and Jan Svartvik (eds.)

1985 A Comprehensive Grammar of the English Language. London: Longman.

Shopen, Timothy

1971 Caught in the act. CLS 7: 254-263.

Stefanowitsch, Anatol

2000 The English GO-(PRT)-AND-VERB construction. BLS 26: 259-270.

Stefanowitsch, Anatol and Stefan Th. Gries

2003 Collostructions: On the interaction between verbs and constructions. International Journal of Corpus Linguistics 8 (2): 209-243.

Vendler, Zeno

1967 Linguistics in Philosophy. Ithaca, NY: Cornell University Press. 